\title{
Infralimbic Neurotrophin-3 Infusion Rescues Fear Extinction Impairment in a Mouse Model of Pathological Fear
}

\author{
Davide D'Amico 1,2,3,6,7, Thomas Gener ${ }^{1,2,3,6}$, Maria Martínez de Lagrán ${ }^{1,2,3,6}$, Maria V Sanchez-Vives ${ }^{4,5}$, \\ Mónica Santos ${ }^{1,2,3,8,9}$ and Mara Dierssen ${ }^{*, 1,2,3,6,9}$
}

'Systems Biology Programme, Centre for Genomic Regulation (CRG)/Barcelona Biomedical Research Park, Barcelona, Spain; ${ }^{2}$ Universitat Pompeu Fabra (UPF), Barcelona, Spain; ${ }^{3}$ Centro de Investigación Biomédica en Red de Enfermedades Raras (CIBERER), Barcelona, Spain; ${ }^{4}$ Systems Neuroscience, IDIBAPS (Institut d'Investigacions Biomèdiques August Pi i Sunyer), Barcelona, Spain; ${ }^{5}$ Life \& Medical Sciences, ICREA (Instituciò Catalana de Recerca i Estudis Avançats), Barcelona, Spain; 'B Barcelona Institute for Science and Technology (BIST), Barcelona, Spain

\begin{abstract}
The inability to properly extinguish fear memories constitutes the foundation of several anxiety disorders, including panic disorder. Recent findings show that boosting prefrontal cortex synaptic plasticity potentiates fear extinction, suggesting that therapies that augment synaptic plasticity could prove useful in rescue of fear extinction impairments in this group of disorders. Previously, we reported that mice with selective deregulation of neurotrophic tyrosine kinase receptor, type 3 expression (TgNTRK3) exhibit increased fear memories accompanied by impaired extinction, congruent with an altered activation pattern of the amygdala - hippocampus - medial prefrontal cortex fear circuit. Here we explore the specific role of neurotrophin 3 and its cognate receptor in the medial prefrontal cortex, and its involvement in fear extinction in a pathological context. In this study we combined molecular, behavioral, in vivo pharmacology and ex vivo electrophysiological recordings in TgNTRK3 animals during contextual fear extinction processes. We show that neurotrophin 3 protein levels are increased upon contextual fear extinction in wild-type animals but not in TgNTRK3 mice, which present deficits in infralimbic long-term potentiation. Importantly, infusion of neurotrophin 3 to the medial prefrontal cortex of TgNTRK3 mice rescues contextual fear extinction and ex vivo local application improves medial prefrontal cortex synaptic plasticity. This effect is blocked by inhibition of extracellular signal-regulated kinase phosphorylation through peripheral administration of SL327, suggesting that rescue occurs via this pathway. Our results suggest that stimulating neurotrophin 3-dependent medial prefrontal cortex plasticity could restore contextual fear extinction deficit in pathological fear and could constitute an effective treatment for fear-related disorders.
\end{abstract}

Neuropsychopharmacology (2017) 42, 462-472; doi:I 0.I 038/npp.2016.154; published online 21 September 2016

\section{INTRODUCTION}

Extinction is a learning process that encodes new valence for the conditioned stimulus through repeated unreinforced reexposure (Quirk and Mueller, 2008; Sotres-Bayon et al, 2007). It offers the therapeutic possibility of diminishing the impact caused by the intrusive recollection of traumatic events (Parsons and Ressler, 2013). However, impaired extinction of fear memories is thought to contribute to the development and persistence of anxiety disorders including phobias, post-traumatic stress disorder and panic disorder (PAND). Specifically, PAND patients show exaggerated and

*Correspondence: Professor M Dierssen, Systems Biology Programme, Center for Genomic Regulation, (CRG)/Barcelona Biomedical Research Park, C/ Dr Aiguader 88, Barcelona 08003, Spain, Tel: +34 933।60 I40, Fax+349331600 99, E-mail: mara.dierssen@crg.es

${ }^{7}$ Current address: ZeClinics SL, E-0800। Barcelona, Spain.

${ }^{8}$ Current address: Institute of Biology, Otto-von-Guericke-University. Magdeburg, D39120 Magdeburg, Germany.

${ }^{9}$ These authors contributed equally to this work.

Received 26 January 2016; revised 5 August 2016; accepted 7 August 2016; accepted article preview online 18 August 2016 pathological fear (Lissek et al, 2005; Lissek et al, 2009) that is resistant to extinction (Michael et al, 2007).

The medial prefrontal cortex (mPFC) and its interactions with the amygdala and the hippocampus are critical in the extinction of fear associations (Sierra-Mercado et al, 2011). Specifically, the infralimbic (IL) region of the mPFC is important in tonic inhibition of subcortical structures and emotional responses-such as fear, and is the core region in extinction of conditioned fear (Milad and Quirk, 2002; Vidal-Gonzalez et al, 2006). Fear extinction involves physiological mechanisms or neural plasticity in the mPFC including protein synthesis (Santini et al, 2004), glutamatergic receptor activation (Sotres-Bayon and Quirk, 2010) and synaptic long-term potentiation (Peters et al, 2010). Brainderived neurotrophic factor (BDNF) and neurotrophin 3 (NT3) have been firmly established as regulators of neuronal development and synaptic plasticity in the mammalian brain (McAllister et al, 1999). In the adult brain, neurotrophic tyrosine kinase receptor, type 3 (NTRK3, aka TrkC) promotes synaptogenesis by inducing differentiation of functional excitatory presynaptic terminals (Takahashi et al, 2011). Recently, it was shown that this effect is 
modulated by its cognate ligand-NT3 (AmmendrupJohnsen et al, 2015; Han et al, 2016). In another study, establishment of dendritic arborization of Purkinje cells of the cerebellum depends on a correct balance between NT3 and TrkC levels (Joo et al, 2014).

Brain-derived neurotrophic factor (BDNF) has been shown to potentiate extinction memory when infused into the $\mathrm{mPFC}-\mathrm{IL}$ brain region (Peters et al, 2010). We have explored the role of NT3 and its high-affinity receptor NTRK3 (aka TrkC). NTRK3 gene has been associated with PAND and other psychiatric conditions in human genetic studies (Armengol et al, 2002; Gratacos et al, 2001; MuinosGimeno et al, 2009). We previously demonstrated that transgenic mice overexpressing the full-length NTRK3 (TgNTRK3 mouse model) recapitulate PAND phenotypes (Dierssen et al, 2006). Importantly, TgNTRK3 mice show fear memories that are resistant to contextual extinction, along with an altered neuronal activation in the hippocampus-amygdala-mPFC fear circuit (Santos et al, 2013). This provides strong face validity, since PAND patients also show exaggerated and pathological fear (Lissek et al, 2005; Lissek et al, 2009) that is resistant to extinction (Michael et al, 2007). These patients also show structural and functional alterations in regions of the limbic system and cingulated cortex (reviewed in Santos et al, 2015).

Here we explored the role of NT3/TrkC system in contextual fear extinction and the possibility of targeting the NT3 system for alleviating pathological fear in this PAND model. We found that local NT3 infusion rescued mPFC-IL long-term potentiation (LTP) and the ability of TgNTRK3 animals to extinguish fear memory. These effects were blocked by inhibition of phosphorylation of extracellular signal regulated kinase (ERK) through perypheral administration of SL327, suggesting that they occur via ERK pathway. Our work shows for the first time the involvement of NT3/TrkC and ERK signaling in the modulation of pathological fear and highlights a potential novel pathway to target in fear-related disorders.

\section{MATERIALS AND METHODS}

\section{Animals}

Young adult (two to four months old) male wild-type (WT) and TgNTRK3 (Dierssen et al, 2006) mice were used in all experiments. The colony was maintained by crossing TgNTRK3 males with C57Bl/6SJL females (purchased from Jackson laboratory, Bar Harbor, Maine, USA). A double transgenic line was generated-Thy1-YFP/NTRK3-by crossing TgNTRK3 animals with the commercially available transgenic B6.Cg-Tg(Thy1-YFPH)2Jrs/J (Feng et al, 2000; Jackson laboratory). Double mutants express the human NTRK3 transgene and the reporter gene yellow fluorescent protein (YFP) under the Thy1 promoter. WT littermates served as controls for all the experiments. Pups were weaned at postnatal day 21 and group housed (3-5 animals) in standard laboratory cages filled with sawdust. Mice were maintained at controlled temperature, on a $12 \mathrm{~h}$ light $/ 12 \mathrm{~h}$ dark cycle, with standard food pellets (Mucedola, MI, Italy) and water ad libitum. All behavioral experiments were conducted during the light period of the light/dark cycle. All experiments were performed in accordance with the
European Communities Council Directive (86/609/EEC) and all the procedures were approved by the local ethical committee (CEEA: JMC-07-1001-MDS; MDS-08 1116; MDS-09-1165; MDS-13-1492).

\section{Contextual Fear Conditioning and Extinction}

The contextual fear conditioning protocol was performed in a fear conditioning apparatus (StartFear, Panlab Harvard Apparatus, Barcelona, Spain) and freezing behavior was automatically recorded using commercial software (FREEZING, Panlab Harvard Apparatus). Mice were presented with five random aversive foot shocks (US) in an initially neutral context (CS) to elicit a conditioned freezing response. On day 1 , animals were placed in the testing chamber for a three minutes ( $\mathrm{min}$ ) habituation session. On day 2, mice were trained in the same chamber in a five min session composed of two min exploration, followed by five US presentations (foot shock: $2 \mathrm{~s}, 0.2 \mathrm{~mA}$ ) separated by a variable inter-trial interval (ITI, between 15-60 s); mice remained in the chamber for additional $30 \mathrm{~s}$ after the last US presentation. Freezing behavior was measured in the $15 \mathrm{~s}$ period after each shock. The total time spent freezing in the $15 \mathrm{~s}$ interval was quantified and presented as percentage of freezing time.

Twenty-four hours after training mice underwent extinction of contextual conditioned fear (day 3). The extinction phase consisted of one session of six trials (E1-E6), each lasting two min and separated from the next by 1-h interval. The following day (day 4) mice were tested for fear extinction memory in the same context for two min. A control group was also included in the study that did not go through the fear conditioning and extinction paradigms, but was exposed to the behavioral room and handled by the researcher.

Data were analyzed using repeated measures two-way ANOVA with genotype as between-subject factor and trials (E1-E6) as within-subject factors (fear conditioning and fear extinction training sessions) and two-way ANOVA with genotype and treatment as factors (test session). $P<0.05$ was assumed as critical value for significance throughout the study. Statistical analyses were performed using SPSS 22.0 package.

\section{Protein Extraction}

WT and TgNTRK3 mice were anaesthetized (mixture of $1.0 \mathrm{mg} / \mathrm{kg}$ medetomidine and $0.1 \mathrm{mg} / \mathrm{kg}$ ketamine) and intracardiac perfused with phosphate buffered saline (PBS, $0.1 \mathrm{M} \mathrm{pH} \mathrm{7.6)}$ to clean excess blood. Brains were removed and the hippocampus and PFC regions dissected and frozen in dry ice. For total protein extraction, tissue was homogenized with syringe and $20 \mathrm{G}$ needle in $150 \mu \mathrm{l}$ of lysis buffer (137 mM sodium chloride, $20 \mathrm{mM}$ Tris-hydroxide chloride $\mathrm{pH} 8,1 \%$ nonidet $\mathrm{P} 40$ and $10 \%$ glycerol) with protease (1 complete tablet and $1 \mathrm{mM}$ phenylmethylsulfonyl fluoride) and phosphatase ( $1 \mathrm{mM}$ sodium fluoride and $1 \mathrm{mM}$ sodium orthovanadate) inhibitors. Samples were centrifuged at $13200 \mathrm{rpm}$ for $30 \mathrm{~min}$ at $4{ }^{\circ} \mathrm{C}$ and the supernatants collected and stored at $-80^{\circ} \mathrm{C}$. Protein quantification was performed with the BCA protein assay kit (Thermo Scientific, Rockford, IL, USA). 


\section{Western Blot for TrkC Receptor}

Forty microgram of PFC total protein extracts from WT and TgNTRK3 were loaded in NuPAGE $4-12 \%$ Bis-Tris polyacrylamide gels (NP0336, Novex, Life Technology, Invitrogen, Carlsbad, CA, USA) and bands separated by electrophoresis. Proteins were transferred to nitrocellulose membranes using the iBlot Gel Transfer Stacks kit (IB301001, Novex) and incubated in Odyssey blocking buffer (Novex). Membranes were incubated with primary goat anti-TrkC antibody (1:1000, 07-226, Upstate, Temecula, CA, USA) for $1 \mathrm{~h}$ and with mouse anti-GAPDH (1:4000, MAB374, Millipore, Temecula, CA, USA) for $15 \mathrm{~min}$, at room temperature (RT). Finally, membranes were incubated with the corresponding secondary antibodies polyclonal rabbit anti-goat Alexa Fluor 680LT (1:6000, A-21088, Invitrogen) and donkey anti-mouse IRDye 800CW (1:15000, 926-32212, LI-COR) for $1 \mathrm{~h}$ at RT. Detection was performed using Odyssey infrared scanner (LiCor) and bands were quantified using Odyssey software. Data are reported as percentage of TrkC/GAPDH ratio as referred to control littermates within each genotype. Statistical analysis was performed using 2-way ANOVA with genotype and treatment as factors (Control vs Fear conditioning vs Extinction).

\section{Quantification of NT3 Levels by Enzyme-Linked Immunosorbent Assay (ELISA)}

Quantification of NT3 levels was performed using the Mouse NT3 ELISA kit (BEK-2079, Biosensis, Thebarton, Australia). To detect a reliable amount of NT3, $300 \mu \mathrm{g}$ of total protein extract from WT and TgNTRK3 hippocampus and PFC brain homogenates were used. Briefly, samples and protein standards were loaded in pre-coated 96-well microplates and incubated with mouse anti-NT3 primary antibody, overnight at $4{ }^{\circ} \mathrm{C}$ with agitation. Followed by incubation with biotinylated anti-mouse for $2 \mathrm{~h}$ at $\mathrm{RT}$ with agitation. Development was performed using the avidin-biotinperoxidase $(\mathrm{ABC})$ complex for $1 \mathrm{~h}$ at $\mathrm{RT}$ with agitation. Finally, incubation with TMB color developing agent was performed for ten $\mathrm{min}$ and the reaction was stopped with TMB stop solution. The amount of NT3 was quantified by reading absorbance at $450 \mathrm{~nm}$ on a Versa max plate reader (Molecular Devices, Sunnyvale, CA, USA).

Data were reported as the percentage of NT3 levels relative to control littermates within each genotype. Statistical analysis was performed using two-way ANOVA with genotype and treatment as factors (Control vs Fear conditioning $v s$ Extinction).

\section{Immunofluorescence}

One hour after the last extinction trial (E6), Thy1-YFP/WT and Thy1-YFP/NTRK3 animals were anesthetized and intracardiac perfused with PBS followed by fixation with $4 \%$ paraformaldehyde (PFA) in PBS. Brains were removed and kept in $4 \%$ PFA for $24 \mathrm{~h}$ at $4{ }^{\circ} \mathrm{C}$ for post-fixation, and then passed to a solution of $30 \%$ sucrose in PBS for 2 days at $4{ }^{\circ} \mathrm{C}$. Free floating coronal sections $(40 \mu \mathrm{m})$ were obtained using a Vibrotome (VT1000S, Leica Microsystems, Wetzlar, Germany).
Free floating brain sections were permeabilized with $0.3 \%$ Triton X-100 in PBS for 30 min and blocked in 3\% BSA/0.3\% Triton X-100 in PBS for $1 \mathrm{~h}$, at RT. Subsequently, sections were incubated overnight at $4{ }^{\circ} \mathrm{C}$ with primary rabbit polyclonal anti-c-Fos antibody (1:500, H-125, Santa Cruz Biotechnology) or mouse anti-pERK1/2 (1:1500, SAB1305560, Sigma-Aldrich, Saint Louis, MO, USA). Secondary antibody alexa fluor 594 goat anti-rabbit IgG (1:1000 Invitrogen) was incubated for $1 \mathrm{~h}$ at RT. Sections were mounted on glass slides and nuclei stained with Vectashield with DAPI (Vector Laboratories).

\section{Quantification of c-Fos-, pERK- and YFP-Positive Cells}

Analysis of Thy1-YFP/c-Fos and Thy1-YFP/pERK doublepositive neurons was performed in one of every sixth section of each animal, covering the entire prefrontal cortex (bregma 2.10 through 1.54). For each section, images were captured with a confocal microscope (TCS SP5, Leica Microsystems) using a $40 \times$ objective. Ten $\mu \mathrm{m}$-thick $\mathrm{z}$-stacks were captured that included one picture centered in the $\mathrm{MPFC}$-IL region of the PFC and $\mathrm{z}$-maximum projections for each image was obtained by IMAGEJ 1.421 software. The area of interest was delimited and the number of YFP-, c-Fos- and pERKpositive neurons was quantified. Data were presented as the percentage of double-positive neurons per total number of YFP-positive neurons analyzed. Results are reported as mean \pm SEM and analyzed using Student's $t$-test.

\section{Stereotaxic Surgery}

Animals were injected subcutaneously with $0.05 \mathrm{mg} / \mathrm{kg}$ buprenorfine and $15 \mathrm{~min}$ later were deeply anesthetized with an i.p. mixture of $1 \mathrm{mg} / \mathrm{kg}$ medetomidine $/ 75 \mathrm{mg} / \mathrm{kg}$ ketamine. The head was fixed in a stereotaxic apparatus (Just for Mice Standard Stereotaxic Instrument, Harvard Apparatus, Holliston, MA, USA) and two holes were opened in the skull corresponding to the $\mathrm{mPFC}$-IL region, following stereotaxic coordinates (Paxinos and Franklin, 2001): antero-posterior +2.0 , medio-lateral \pm 0.50 , dorso-ventral -1.2 . Bilateral cannulae (0.5 outer/0.25 inner diameter, AISI 304 Unimed, Lausanne, Switzerland) were implanted and fixed (Simplex Rapid, Kemdent, UK). Animals were awaked with antisedan (i.p. $2 \mathrm{mg} / \mathrm{kg}$ ). For three days following surgery mice were administered buprenorphine (i.p. $0.05 \mathrm{mg} / \mathrm{kg}$ ) for analgesia and checked for general health status. Behavioral procedures were performed at least seven days after surgery. Histological confirmation of the implantation site was performed for all animals at the end of behavioral protocol. Animals where cannulae were not correctly located in the mPFC-IL were excluded from the study (WT-saline $n=1$, TgNTRK3-saline $n=1$ and TgNTRK3-NT3 $n=1)$.

\section{Intra-mPFC NT3 Administration}

Animals were fear-conditioned and subsequently submitted to the fear extinction-training paradigm (as described above). One hour after the last trial of extinction training, recombinant human NT3 (C079, Novoprotein, Shanghai, China), $0.75 \mu \mathrm{l}$ per side from a $1 \mathrm{mg} / \mathrm{ml}$ solution or saline, was infused in the mPFC-IL. The infusion was performed in manually immobilized animals using an internal 
micro-cannula (0.2 outer/0.09 inner diameter, AISI 3161, Unimed) connected through a $15 \mathrm{~cm}$ long PVC tube $(0.25$ inner diameter, F117952, Gilson) to a $5 \mu$ l syringe (Model 75, Hamilton). The internal cannula was introduced into the implanted one and exiting internally into the brain for $1 \mathrm{~mm}$. The infusion was performed at a rate of $250 \mathrm{nl} / \mathrm{min}$. After the infusion, animals were returned to their home cage. For ERK phosphorylation experiment, the specific inhibitor SL327 (50 mg/kg, S4069, Sigma-Aldrich) or saline was peripherally administrated to mice immediately after the last trial of extinction training, followed $1 \mathrm{~h}$ later by NT3 infusion into the MPFC-IL (as described before).

Data were analyzed using repeated measures two-way ANOVA with genotype and treatment as between-subject factors, and extinction trials (E1-E6) as within-subject factors (for extinction training). A two-way ANOVA with genotype and treatment as factors was used to analyze data from extinction test.

\section{Slice Preparation}

After decapitation, the brain was quickly removed and placed on ice-cold cutting solution following (Aghajanian and Rasmussen, 1989); in mM: 252 sucrose, $2.5 \mathrm{KCl}, 3 \mathrm{MgSO}_{4}$, $1.25 \mathrm{NaHPO}_{4}, 1 \mathrm{CaCl}_{2}, 26 \mathrm{NaHCO}_{3}, 10$ dextrose and gassed with $95 \% \mathrm{O}_{2}, 5 \% \mathrm{CO}_{2}$ to a final $\mathrm{pH}$ of 7.4 . Coronal slices (300 $\mu \mathrm{m}$ thick) including the infralimbic cortex (Paxinos and Franklin, 2001) were obtained with a vibratome (Leica, series 1000 classic, Nussloch, Germany), placed in an interface style recording chamber (Fine Science Tools, Foster City, CA) and bathed in artificial cerebrospinal fluid (ACSF) containing (in mM): $124 \mathrm{NaCl}, 2.5 \mathrm{KCl}, 1 \mathrm{MgSO}_{4}, 1.25 \mathrm{NaHPO}_{4}, 2.5$ $\mathrm{CaCl}_{2}, 26 \mathrm{NaHCO}_{3}, 10$ dextrose and aerated with $95 \% \mathrm{O}_{2}$, $5 \% \mathrm{CO}_{2}$ to a final $\mathrm{pH}$ of 7.4. Bath temperature was maintained at $32-34^{\circ} \mathrm{C}$.

\section{Electrophysiological Recordings}

Field postsynaptic potentials (fPSPs) were recorded from layer $\mathrm{V}$ of the mPFC-IL in response to stimulation of layer II/III of the same region (as in Marek et al, 2011). Unfiltered recordings were obtained by means of saline-filled pipettes (impedance 1-2 M $\Omega$ ) through a Neurolog system amplifier (Digitimer, Letchworth Garden City, UK). Electrical stimuli were delivered by means of a concentric bipolar electrodes (platinum-iridium; FHC, Bowdoin, ME, USA) with a stimulus intensity adjusted such that yielded a halfmaximal response $(100-150 \mu \mathrm{A} ; 0.1 \mathrm{~ms}$ duration). For each slice, after determining a stable baseline, paired-pulse protocols were given with an interval of $50 \mathrm{~ms}$ between pulses. To quantify paired-pulse facilitation (PPF) the response was represented as the ratio between both stimuli fPSP2/fPSP1. After $15 \mathrm{~min}$ baseline records (pulse at $0.016 \mathrm{~Hz}$ ), LTP was induced using a HFS protocol (4 episodes of $1 \mathrm{~s}$ at $100 \mathrm{~Hz}$ ) and then recorded for $60 \mathrm{~min}$ (pulse at $0.016 \mathrm{~Hz}$ ). The amplitude of each fPSPs was quantified. The fiber volley was not systematically measured. Recordings were digitized and acquired using Spike2 (Cambridge Electronic Design, Cambridge, UK). For NT3 application experiments the same protocol was used and the drug $(1 \mathrm{mg} / \mathrm{ml})$ was applied in the bath for $20 \mathrm{~min}$ before starting the recordings. Data analysis was carried out with
Spike2 using a custom made script. Statistical analysis was performed using repeated measures two-way ANOVA with genotype and time as factors for LTP and Student $t$-test for PPF.

\section{RESULTS}

\section{Acquisition of Contextual Fear Conditioning and Extinction Regulates Expression Levels of NT3 and TrkC}

To address the potential involvement of changes in the NT3/ TrkC system in fear extinction processes we first quantified the NT3 and TrkC protein levels in the PFC of WT and TgNTRK3 animals. Material was obtained from controls and from animals sacrificed $1 \mathrm{~h}$ after fear conditioning acquisition and $1 \mathrm{~h}$ after the last extinction acquisition trial (Figure 1a). WT and TgNTRK3 animals showed similar increase in the percentage of freezing levels in the contextual fear conditioning paradigm (Figure 1b, trial effect $\mathrm{F}_{(2,20)}=7.781 p=0.002$ ). As previously shown (Santos et al, 2013) we observed impaired acquisition of fear extinction in TgNTRK3 animals, as compared with WT (Figure 1c, trial $\mathrm{x}$ genotype effect $\mathrm{F}_{(5,45)}=2.462 p=0.047$ ).

At the molecular level, WT and TgNTRK3 animals showed no differences in the basal NT3 and TrkC expression levels in the PFC. Interestingly, both fear acquisition and fear extinction significantly increased the expression of TrkC in both WT and TgNTRK3 mice, as compared with control animals of the corresponding genotype (Figure 1d and e; treatment effect $\left.\mathrm{F}_{(2,30)}=7.206 p=0.003\right)$. However, upon both fear acquisition and fear extinction, NT3 levels were increased in WT, but not in TgNTRK3 animals, as compared with control values (Figure 1f; genotype $\mathrm{x}$ treatment interaction $\mathrm{F}_{(2,26)}=4.01 p=0.030$; Bonferroni post hoc: WT-control vs TgNTRK3-control $p=1.0$; WT-FC vs TgNTRK3-FC $p=0.004$; WT-Ext vs TgNTRK3-Ext $p=0.001)$. The increase in NT3 and TrkC levels upon fear acquisition training and extinction training in WT animals suggests a role for these molecules in fear processing. Moreover, data also shows that NT3/TrkC pathway is dysregulated in the $\operatorname{TgNTRK} 3$ mouse model.

In the hippocampus, we quantified the levels of NT3 in WT and TgNTRK3 animals at basal, after fear conditioning and fear extinction (Figure 1g). We observed an increase in the levels of NT3 in both WT and TgNTRK3 animals after fear extinction (treatment effect, $\mathrm{F}_{(2,26)}=5.50 p=0.010$ ), though no differences in NT3 levels were observed between genotypes.

\section{Impaired ERK Activation in Pyramidal mPFC-IL Neurons of TgNTRK3 Mice}

ERK activation, which can be triggered by the NT3/TrkC intracellular signaling cascade ( $\mathrm{Li}$ et al, 2014; Reichardt, 2006), is necessary for successful fear extinction in rodents (Fischer et al, 2007; Tronson et al, 2009). We therefore reasoned that altered $\mathrm{NT} 3 / \mathrm{TrkC}$ function might disturb fear extinction memory in TgNTRK3 mice due to impaired ERK signaling. We analyzed ERK phosphorylation levels in excitatory neurons of mPFC-IL of TgNTRK3 and WT mice $1 \mathrm{~h}$ after the last fear extinction trial, using the double transgenic line Thy1-YFP/NTRK3. No genotype 
differences were found in the total number of excitatory (YFP-positive) neurons in the mPFC-IL (Figure 2a and b and Figure $2 \mathrm{e}$ and $\mathrm{f} ; t_{(9)}=-0.913 p=0.38$ and $t_{(9)}=1.427$ $p=0.19$, respectively). However, the number of pERKpositive cells and YFP/pERK double-positive neurons was significantly reduced in $\operatorname{TgNTRK3}$ as compared with WT

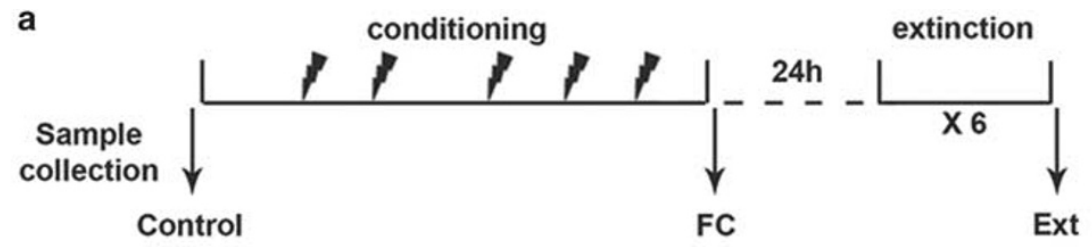

b

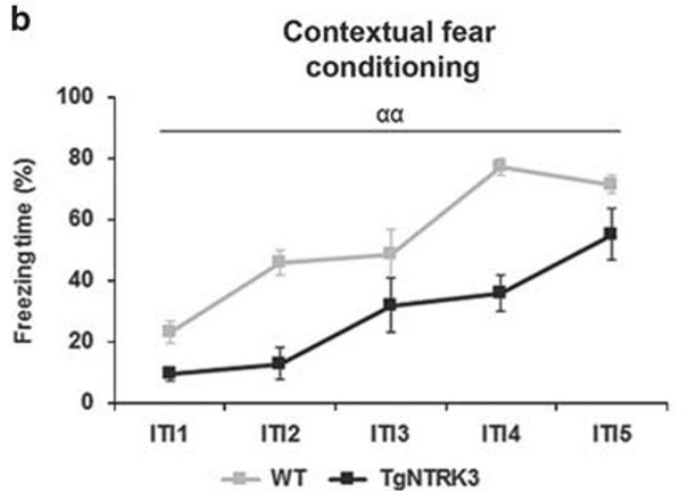

d

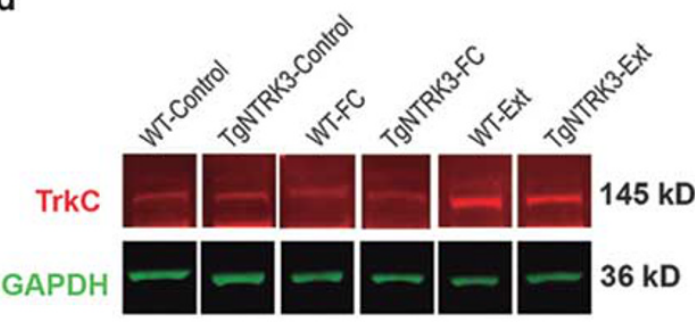

$\mathbf{f}$

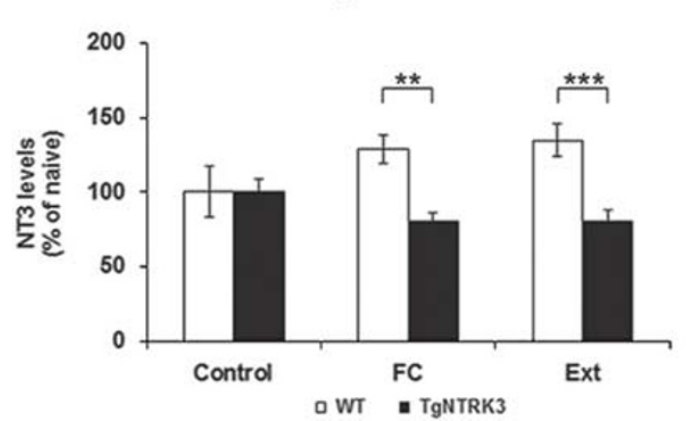

C
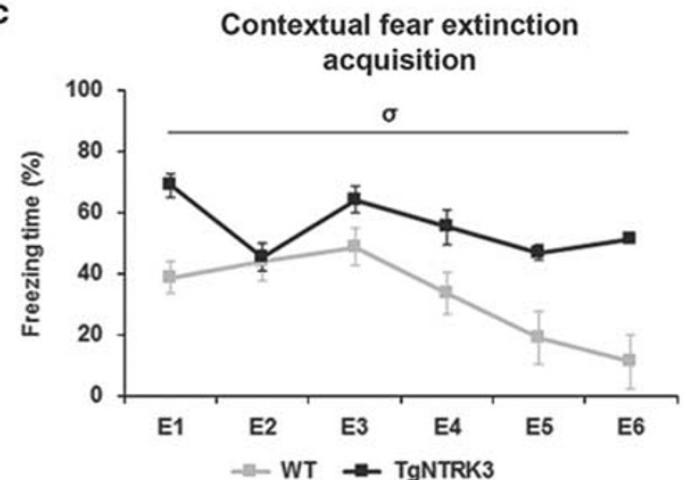

e

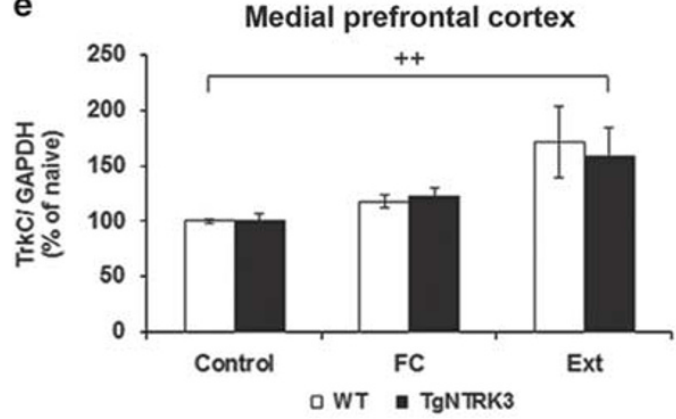

g

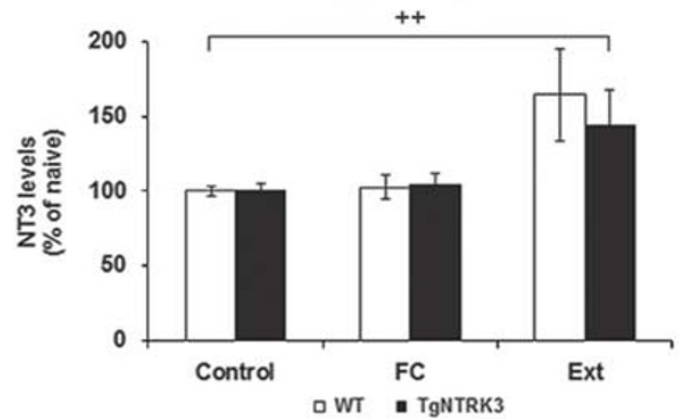

Figure I TgNTRK3 mice show impairment in NT3 expression levels upon fear conditioning and extinction. (a) Schematic representation of experimental setup. WT and TgNTRK3 mice submitted to contextual fear conditioning and within-session extinction protocols. One hour after the last trial of extinction training mice were sacrificed and the prefrontal cortex and hippocampus regions dissected. Control animals were sacrificed without any fear conditioning or extinction protocols. (b) In the contextual fear conditioning, TgNTRK3 and WT animals showed an increase in the percentage of freezing levels along the session. (c) In the fear extinction acquisition session, a reduction in freezing time is observed along extinction trials in WT, but not TgNTRK3 animals (repeated measures two-way ANOVA, WT $n=5, \operatorname{TgNTRK3} n=6$ ). (d) Representative Western blot scans of TrkC full-length isoform and GAPDH housekeeping protein in the prefrontal cortex brain region. (e) Quantification of TrkC/GAPDH expression ratio. Upon fear conditioning and extinction paradigms the levels of TrkC in the PFC of both WT and TgNTRK3 animals are increased (two-way ANOVA, $n=6$ per group). (f) Determination of NT3 levels by ELISA. In the prefrontal cortex, after fear conditioning and fear extinction the levels of NT3 are higher in WT than in TgNTRK3 animals (two-way ANOVA, $n=5$ per group). (g) In the hippocampus, NT3 levels are increased in both WT and TgNTRK3 animals after fear extinction protocol. FC, fear conditioning group; Ext, fear extinction group; EI-E6, extinction trials; GAPDH, glyceraldehyde 3-phosphate dehydrogenase; NT3, neurotrophin type 3; TrkC, tropomyosin kinase receptor, type 3; TgNTRK3, transgenic mice overexpressing NTRK3; WT, wild type. Trial effect, ${ }^{\alpha \alpha} p<0.01$; trial $x$ genotype interaction, ${ }^{\sigma} p<0.05$; treatment effect, ${ }^{++} p<0.0$ I; Bonferroni post hoc comparisons, $* * * 0.0$ I, $* * * * x<0.00$ I. 


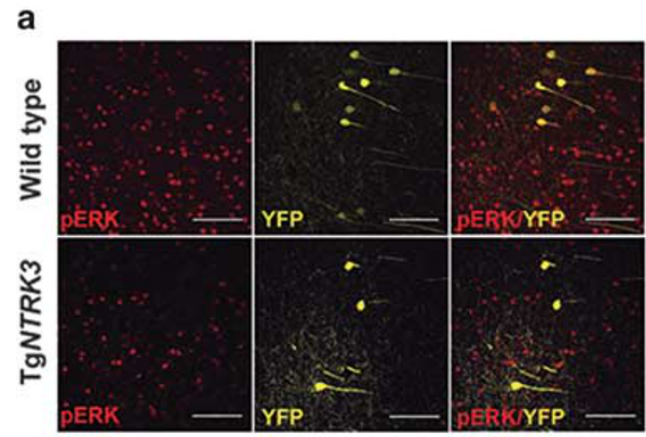

IL pERK excitatory neurons

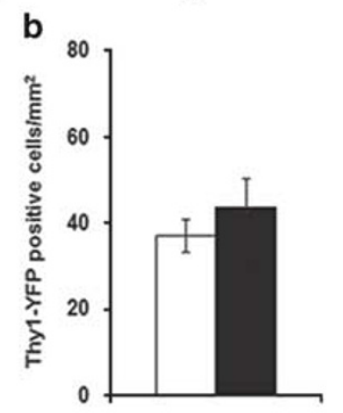

IL c-Fos excitatory neurons
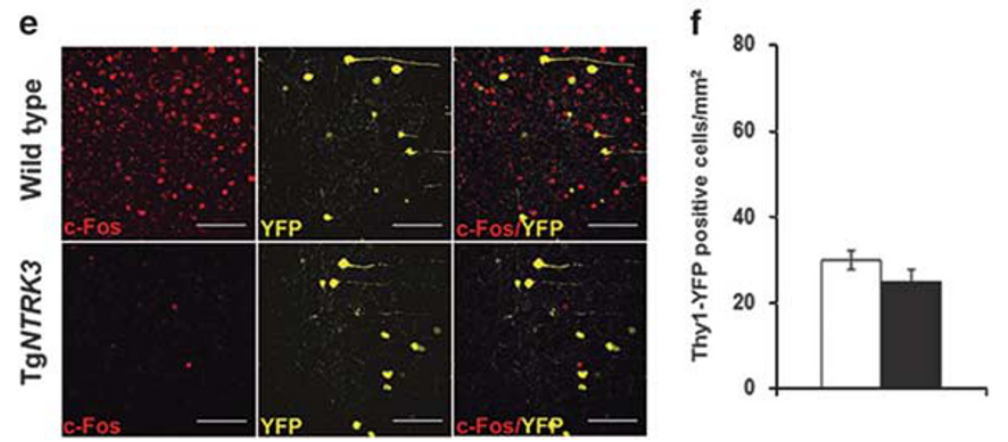

C

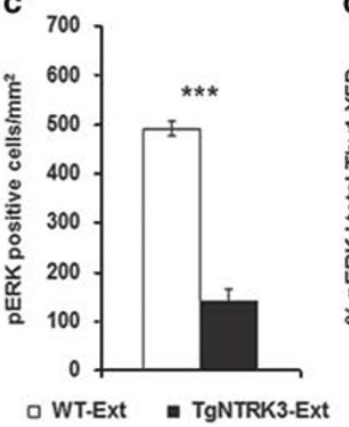

d

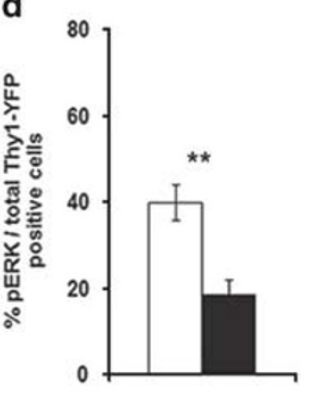

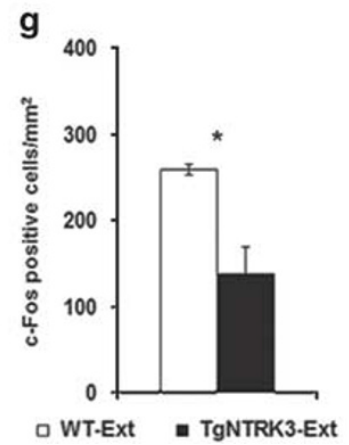

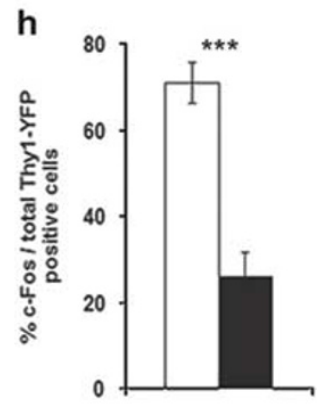

Figure 2 TgNTRK3 show impaired ERK activation and impaired neuronal activation of excitatory neurons in the mPFC-IL region, upon fear extinction acquisition. (a) Representative photomicrographs of pERK immunofluorescence and Thyl-YFP positive neurons in the IL region of the mPFC. Quantification of (b) Thyl-YFP-, (c) pERK- and (d) pERK/YFP double-positive neurons in the IL of WT and TgNTRK3 animals after fear extinction. TgNTRK3-Ext animals showed a reduction in the percentage of pERK-positive excitatory cells as compared with WT-Ext animals (Student's $t$ test; WT-Ext $n=5$; TgNTRK3-Ext $n=5$ ). (e) Representative photomicrographs of c-Fos immunofluorescence and Thyl-YFP positive neurons in the IL region of the mPFC. Quantification of ( $f$ ) Thyl-YFP-, (g) c-Fos- and (h) c-Fos/YFP double-positive neurons in the IL of WT and TgNTRK3 animals after fear extinction. TgNTRK3-Ext animals showed a remarkable reduction in the percentage of activated excitatory neurons, as compared with WT-Ext animals (Student's $t$ test, $n=5 / 6$ ). Ext, fear extinction group; IL, infralimbic; pERK, phosphorylated extracellular signal regulated kinase; TgNTRK3, transgenic mice overexpressing NTRK3; Thyl, thymus cell antigen I; WT, wild type; YFP, yellow fluorescent protein. Scale bar $=100 \mu \mathrm{m}$. Student's $t$ test, $* p<0.05, * * p<0.01, * * * 2<0.001$.

animals (Figure 2a, c and d; pERK, $t_{(9)}=12.483 p=5.5 \mathrm{E}^{-07}$; $\left.\mathrm{YFP} / \mathrm{pERK} t_{(9)}=3.80 \quad p=0.004\right)$. Moreover, upon fear extinction the number of c-Fos-positive cells and YFP/cFos double-positive neurons in the mPFC-IL was significantly reduced in $\operatorname{TgNTRK3}$ animals compared with WT (Figure 2e, g and h; c-Fos, $t_{(4)}=3.705 p=0.018$; YFP/c-Fos, $\left.t_{(9)}=6.24 p=1.5 \mathrm{E}^{-04}\right)$. We suggest that disturbance of ERK signaling may constitute a mechanism of pathological fear directly resulting from changes in the NT3/TrkC signaling pathway in the mPFC-IL of TgNTRK3 mice.

\section{Infusion of NT3 in mPFC-IL Rescues Fear Extinction Impairment in TgNTRK3 Mice via ERK Signaling Pathway}

To determine whether the impairment of fear extinction in TgNTRK3 is the result of a deficit of local NT3 during fear memory formation, we infused NT3 in the MPFC-IL region $1 \mathrm{~h}$ after the last extinction trial and analyzed fear extinction $24 \mathrm{~h}$ later hence targeting the consolidation phase of extinction memory (Figure 3a). WT and TgNTRK3 showed an increase in the percentage of freezing levels along fear conditioning trials (Figure $3 \mathrm{~b}$, trial effect $\mathrm{F}_{(4,128)}=13.144$ $p=2.1 \mathrm{E}-08)$. As before, in the extinction session WT animals showed a progressive reduction in their freezing levels along trials, which was not observed for TgNTRK3 animals (Figure 3c, genotype $\mathrm{x}$ trial interaction $\mathrm{F}_{(3,160)}=3.595 p=0.013$; Bonferroni post hoc WT vs TgNTRK3, E1 $p=0.017, \mathrm{E} 4 p=2.4 \mathrm{E}-05$, E5 $p=2.0 \mathrm{E}-05$, $\mathrm{E} 6 p=7.0 \mathrm{E}-06)$. Infusion of NT3 had a differential effect on extinction memory (tested $24 \mathrm{~h}$ later) in each genotype (Figure 3d, genotype $\mathrm{x}$ treatment interaction $\mathrm{F}_{(1,30)}=16.723$ $p=2.9 \mathrm{E}-04)$. Infusion of NT3 to WT animals nonsignificantly increased the freezing response as compared with WT-saline animals (Bonferroni post hoc, WT-saline vs WT-NT3 $p=0.054)$. By contrast, in TgNTRK3 mice NT3 rescued the impaired fear extinction by reducing freezing levels to WT values (Bonferroni post hoc, WT-saline vs TgNTRK3-saline $p=4.3 \mathrm{E}-05, \quad \operatorname{TgNTRK3}$-saline vs TgNTRK3-NT3 $p=7.9 \mathrm{E}-04$, WT-NT3 vs TgNTRK3-NT3 $p=0.46$ ).

We then addressed whether the NT3-mediated rescue of fear extinction impairment in TgNTRK3 animals was mediated through the ERK pathway. A new group of animals was trained in the fear extinction paradigm and immediately after the last extinction trial was administered i.p. with SL327 (a ERK phosphorylation inhibitor) or vehicle, followed by NT3 infusion into the mPFC-IL $1 \mathrm{~h}$ after the last extinction acquisition trial (Figure 4a). Again, we observed that WT and TgNTRK3 animals increased their percentage of freezing 
a

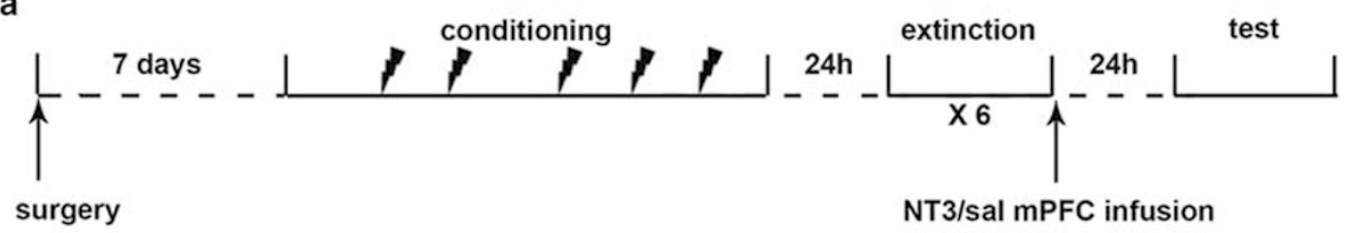

b

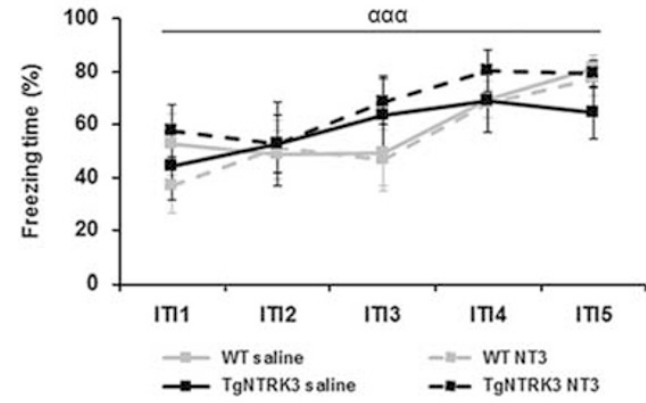

d

d Contextual fear extinction

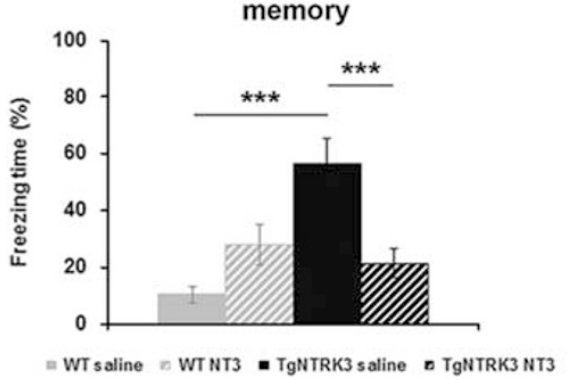

c
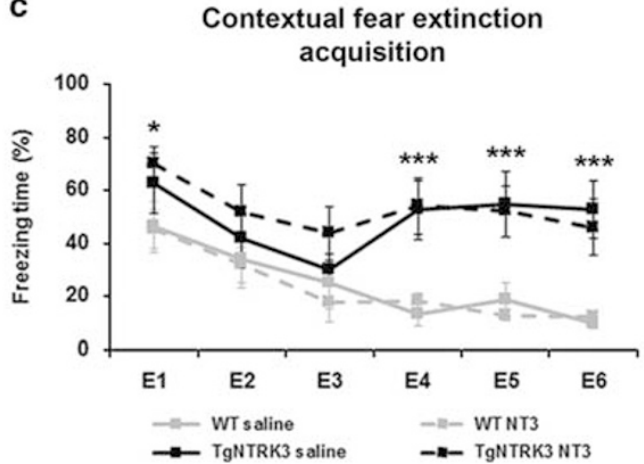

Figure 3 Local NT3 infusion to mPFC-IL rescues fear extinction memory deficit in TgNTRK3 mice. (a) Schematic representation of experimental setup. WT and TgNTRK3 mice were bilaterally implanted with cannulae in the MPFC-IL and submitted to contextual fear conditioning and extinction paradigms. One hour after the last extinction training trial mice were locally infused with saline or NT3 (0.75 $\mu \mathrm{g} /$ side) and tested $24 \mathrm{~h}$ later for fear memory. (b) WT and TgNTRK3 mice increased their percentage of freezing time along fear conditioning sessions (repeated measures two-way ANOVA). (c) In the fear extinction acquisition session, a reduction in freezing time is observed along extinction trials in WT but not TgNTRK3 groups (repeated measures two-way ANOVA). (d) Local infusion of NT3 into mPFC I h after the last extinction training trial, reduced the percentage of freezing time of TgNTRK3 animals when tested $24 \mathrm{~h}$ later, thus rescuing the fear extinction deficit (two-way ANOVA). WT-saline $n=8$, WT-NT3 $n=10$, TgNTRK3-saline $n=7$ and TgNTRK3-NT3 $n=9$. E I-E6, extinction trials I-6; mPFC, medial prefrontal cortex; NT3, neurotrophin type 3; TgNTRK3, transgenic mice overexpressing NTRK3; WT, wild type. Trial effect, ${ }^{\alpha \alpha \alpha} p<0.00$ I; genotype effect, ${ }^{\sigma \sigma \sigma} p<0.00$ I; Bonferroni post hoc comparisons, ${ }^{*} p<0.05$, ${ }^{*} * * * x<0.00$ I.

time along conditioning trials (Figure $4 \mathrm{~b}$; trial effect $\left.\mathrm{F}_{(4,88)}=15.34 p=1.48 \mathrm{E}-09\right)$. Additionally, we again observed a genotype difference in freezing levels during the extinction training paradigm with WT groups reducing freezing and TgNTRK3 groups maintaining high freezing levels (Figure 4c; genotype effect $\mathrm{F}_{(1,}$ 22) $=17.49$ $p=3.9 \mathrm{E}-04)$. NT3 recovered extinction in TgNTRK3, but peripheral administration of SL327 blocked the NT3-induced extinction memory in both WT and TgNTRK3 animals (Figure 4d; treatment effect $\left.\mathrm{F}_{(1,20)}=15.47 p=0.001\right)$. Thus indicating that signaling through the ERK pathway is indispensible for the NT3 dependent consolidation of extinction memory.

\section{Long-Term Potentiation Deficit in mPFC-IL Region of TgNTRK3 Mice is Rescued by Local NT3 Administration}

Finally, we measured LTP (Figure 5a and b) and paired pulse facilitation (PPF) in WT and TgNTRK3 mPFC-IL brain slices. The LTP protocol was carried out in both genotypes by stimulating layers II/III and recording in layer $\mathrm{V}$ of the mPFC-IL (Marek et al, 2011). An early potentiation of the fPSPs was evoked in both genotypes (Figure $5 c$ and e). Following $1 \mathrm{~h}$ of recording, WT animals showed LTP while the averaged PSP's amplitude was back to baseline values in the TgNTRK3. LTP in TgNTRK3 mice was thus significantly reduced with respect to WT (Figure $5 c$, genotype effect $\left.\mathrm{F}_{(1,24)}=31.83 p=8.27 \mathrm{E}-6\right)$. When NT3 was applied to the bath $20 \mathrm{~min}$ before initiating the recordings, LTP was efficiently induced in both WT and TgNTRK3 brain slices, with no significant differences between genotypes (Figure 5e, genotype effect $\left.\mathrm{F}_{(1,18)}=0.11 p=0.7439\right)$, hence rescuing the impaired local LTP in the MPFC-IL brain region of TgNTRK3 animals.

No significant differences were found between WT and TgNTRK 3 animals in short term plasticity in the form of PPF (Figure $5 \mathrm{~d}, t_{(34)}=0.196 p=0.846$ ), and no differences 
a

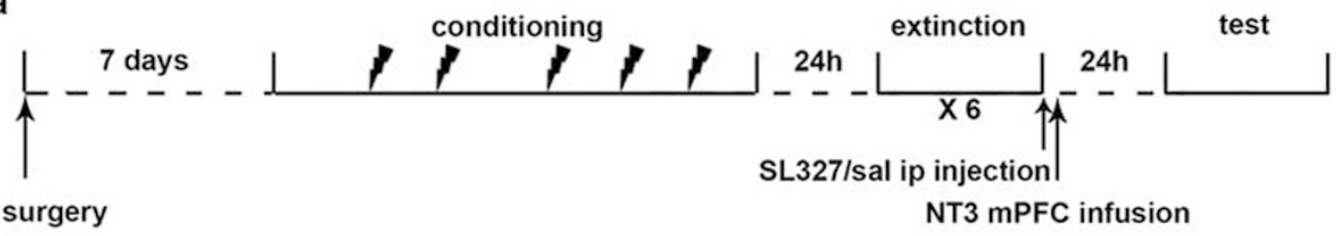

b

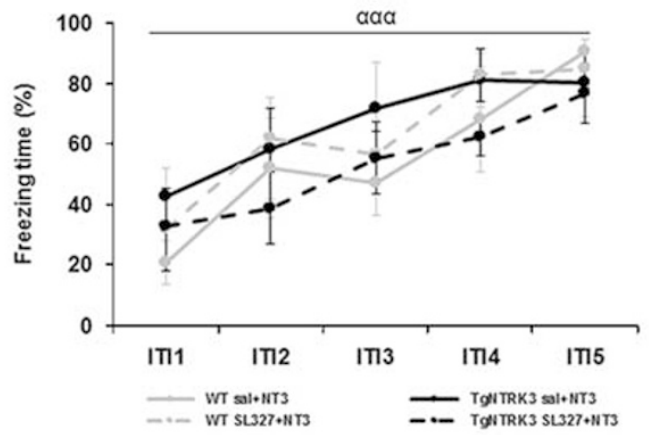

d

\section{Contextual fear extinction} memory

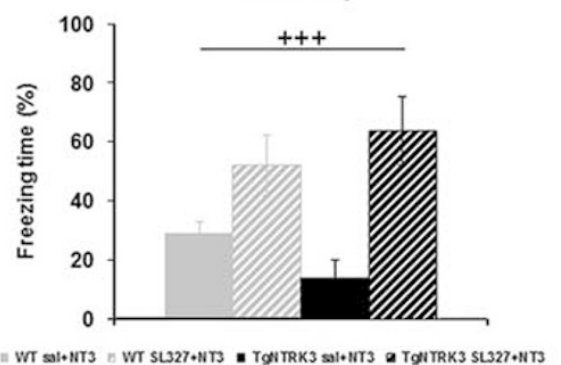

C

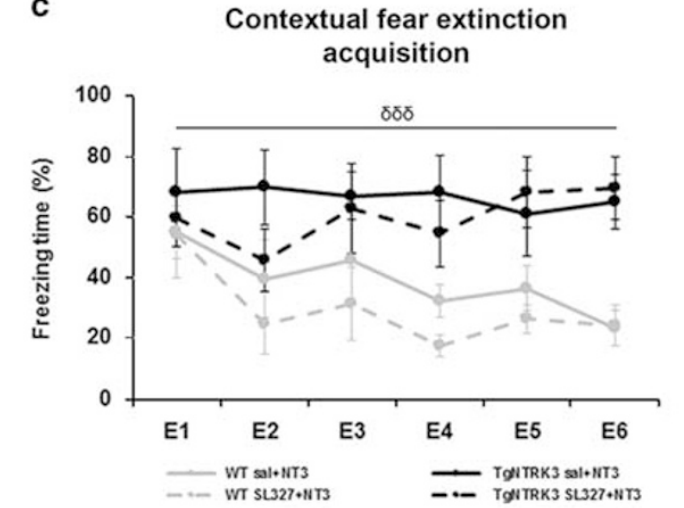

Contextual fear extinction acquisition

Figure 4 Local NT3-mediated rescue of fear extinction memory in TgNTRK3 mice occurs via ERK pathway. (a) Schematic representation of experimental setup. A new group of WT and TgNTRK3 mice were bilaterally implanted with cannulae in the mPFC and submitted to contextual fear conditioning and extinction paradigms. Immediately after extinction training mice were administered i.p. either saline or the ERK phosphorylation inhibitor SL327 (50 mg/kg). One hour later mice were infused with NT3 $(0.75 \mu \mathrm{g} /$ side) and tested $24 \mathrm{~h}$ later for fear memory. (b) WT and TgNTRK3 mice increased their percentage of freezing time along fear conditioning sessions (repeated measures two-way ANOVA). (c) WT, but not TgNTRK3, showed a reduction in freezing time along extinction trials (repeated measures two-way ANOVA). (d) Peripheral administration of SL327 blocked the NT3-dependent reduction of freezing time in WT, but also in TgNTRK3 animals, showing that the NT3 effect is mediated by ERK signaling (two-way ANOVA). WT-sal+NT3 $n=5$, WT-SL327+NT3 $n=6$, TgNTRK3-sal+NT3 $n=6$, TgNTRK3-SL327+NT3 $n=7$. NT3, neurotrophin 3; mPFC, medial prefrontal cortex; WT, wild type; TgNTRK3, transgenic mice overexpressing NTRK3; EI-E6, extinction trials I-6. Trial effect, ${ }^{\alpha \alpha \alpha} p<0.00$ I; genotype effect, ${ }^{\sigma \sigma \sigma} p<0.00$ I; treatment effect, ${ }^{+++} p<0.00$ I.

were detected either upon NT3 application (Figure 5f, $\left.t_{(20)}=1.323 p=0.201\right)$.

\section{DISCUSSION}

Fear extinction is of clinical relevance in maladaptive fear and is the cornerstone of psychological therapy of several anxiety disorders (Parsons and Ressler, 2013). Recent findings in rodents and nonhuman primates showed that modulation of plasticity in the mPFC affects extinction and alters fear output. In humans, patients affected by PAND are unable to extinguish fear memories (Michael et al, 2007) and present neuroanatomical alterations in prefrontal cortical regions (reviewed in Santos et al, 2015). In our previous work, we have shown deficits in extinction of contextual fear memory in TgNTRK3, a mouse model of PAND (Santos et al, 2013). We here report a deficit in the NT3/TrkC-ERK intracellular signaling in this brain region that seems to causally involved in fear extinction impairment in $\operatorname{TgNTRK3}$ mice and can be recovered by local substitution of NT3 to the mutant mPFC. Targeting plasticity in the mPFC could constitute a therapeutic approach in the treatment of anxiety disorders with deficits in TrkC mediated signaling.

The TgNTRK3 shows a complex pattern of TrkC expression in the brain suggesting that regulatory mechanisms are in place, leading to age-dependent and subtle and region-specific differences in the adult TgNTRK3 animals. TgNTRK3 brains show higher TrkC expression levels than WT at postnatal day 14 pups, which is less pronounced in adult animals (Dierssen et al, 2006). Moreover, adult TgNTRK3 showed higher TrkC expression than WT animals in some brain regions but not in others (Dierssen et al, 2006). In the present study, at baseline no differences were detected 


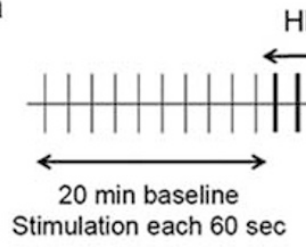

C

WT<smiles>Cc1ccccc1I</smiles>

TgNTRK3
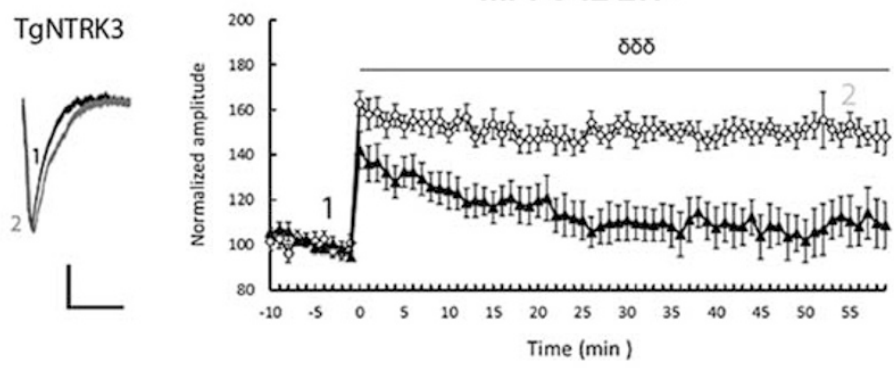

$\infty$ Mean WT $\neq$ Mean TgNTRK3

e $\begin{array}{cc}\text { WT } & \text { TgNTRK3 } \\ +\mathrm{NT3} & +\mathrm{NT3}\end{array}$

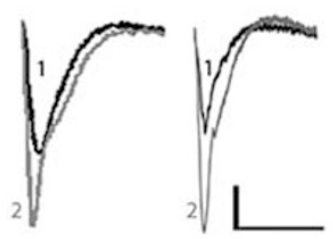

HFS

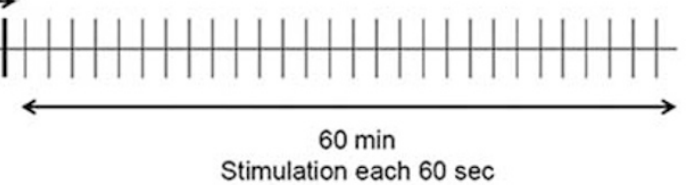

MPFC-IL LTP

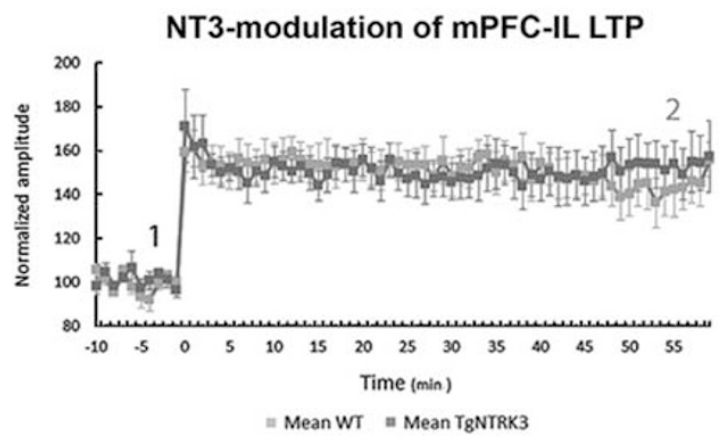

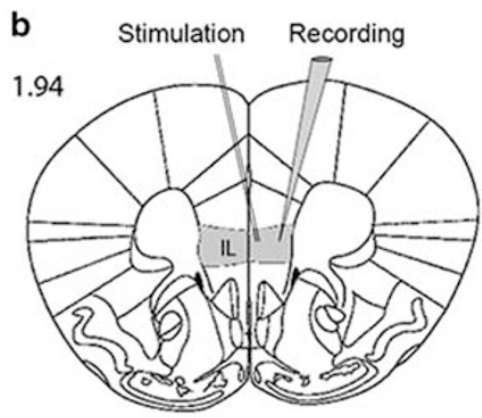

d

MPFC-IL PPF

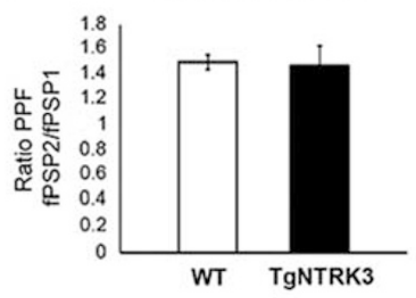

f

NT3-modulation of mPFC-IL PPF

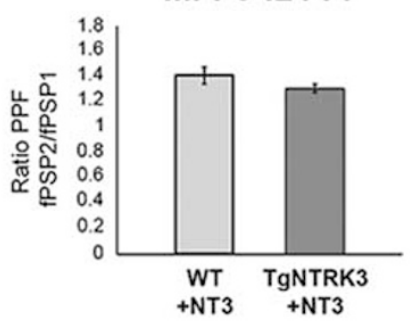

Figure 5 Synaptic plasticity in the mPFC-IL region of TgNTRK3 mice is impaired and restored upon local NT3 infusion. (a) Schematic representation of the LTP protocol. Briefly, all groups received 20 min baseline recording (one stimulation each minute), after which high frequency stimulation was applied (HFS, four episodes of I s at $100 \mathrm{~Hz}$ ), and followed by I h recording (one stimulation each minute). (b) Schematic representation of mPFC-IL stimulation and recording sites. Stimulation electrode in layer II/III, and recording electrode in layer V of mPFC-IL. (c) TgNTRK3 animals showed a deficit in LTP maintenance in the $\mathrm{mPFC}-\mathrm{IL}$ region as compared with WT animals (repeated measures two-way ANOVA with genotype and time points as factors; WT, $n=1$ I slices from a total of five mice; TgNTRK3, $n=15$ slices from a total of seven mice). On the left, examples of WT and TgNTRK3 recordings at time I (baseline, black) and time 2 ( I h after HFS; gray). (d) No differences were observed in PPF between WT and TgNTRK3 (Student's $t$ test; WT, $n=21$ slices from a total of five mice; TgNTRK3, $n=15$ slices from a total of seven mice). (e) Local NT3 application completely rescued LTP in TgNTRK3 slices without affecting WT values (repeated measures two-way ANOVA with genotype and time points as factors; WT, $n=1$ I slices in a total of seven mice; TgNTRK3, $n=9$ slices in a total of six mice). On the left, examples of WT+NT3 and TgNTRK3+NT3 recordings at time I (baseline, black) and time 2 (I h after HFS, gray). ( $f$ ) No differences were observed in PPF between WT+NT3 and TgNTRK3+NT3 (Student's $t$ test; WT, $n=12$ slices from a total of five mice; TgNTRK3, $n=10$ slices from a total of seven mice). LTP, long-term potentiation; mPFC-IL, medial prefrontal cortex-infralimbic; NT3, neurotrophin 3; PPF, paired pulse facilitation; TgNTRK3, transgenic mice overexpressing NTRK3; WT, wild type. Genotype effect ${ }^{\sigma \sigma \sigma} p<0.00$ I.

in TrkC levels between genotypes in PFC. As a result, TgNTRK3 mouse model has a deregulation in TrkC levels across brain regions that may affect plasticity.

First, we observed that TgNTRK3 did not show the extinction-related increase in NT3 levels in the mPFC that we observed in WT animals. Binding of NT3 to its highaffinity TrkC receptor results in intracellular activation of ERK signaling (Li et al, 2014; Reichardt, 2006). Therefore, the NT3 deficit observed in TgNTRK3 animals is likely to result in a deficit in the activation of the NT3/TrkC downstream pathway ERK. Since activation of ERK, as measured by its phosphorylation levels, is associated with successful fear extinction (Fischer et al, 2007; Tronson et al, 2009), reduced ERK phosphorylation in the MPFC-IL of TgNTRK3 in turn may explain the impairment in fear extinction memory observed in the mutant mice. In fact, TgNTRK3 animals showed a reduction in ERK phosphorylation in $\mathrm{MPFC}-\mathrm{IL}$ excitatory neurons along with a reduction in neuronal activation (as measured by c-Fos levels). Excitatory projecting neurons in the mPFC-IL activate the medial intercalated cell cluster, a key inhibitory checkpoint in the amygdala circuit during fear extinction (Sierra-Mercado et al, 2011), thereby playing a central role in signaling 'fear off' (VidalGonzalez et al, 2006). The lack of NT3 upregulation in our 
PAND mouse model is in line with an impairment of such fear-off signals, leading to the observed fear extinction deficit.

Indeed, NT3 infusion into the mPFC-IL region of TgNTRK3 mice during the consolidation phase of fear extinction was sufficient to rescue the impaired fear extinction phenotype. This effect can be attributed to the downstream activation of the ERK signaling, as inhibition of ERK, using the specific inhibitor SL327, blocks NT3mediated extinction in $\operatorname{TgNTRK} 3$ mice.

To evaluate the PFC effects of NT3-induced extinction on synaptic plasticity, we used ex vivo electrophysiology recordings in the mPFC-IL. TgNTRK3 animals showed a deficit in LTP in the MPFC-IL region, confirming reduced synaptic plasticity in this brain region. In agreement with our behavioral observations, the impaired LTP in the mPFC-IL was completely rescued when NT3 was applied to mPFC brain slices of TgNTRK3 mice. The NT3/TrkC pathway actively modulates the glutamatergic system by increasing excitatory currents through the modulation of presynaptic neurotransmitter containing vesicles (Collin et al, 2001). Moreover, exogenous application of NT3 can enhance glutamatergic synaptic transmission via activation of TrkC receptor, by increasing the efficacy of glutamate release (Lessmann, 1998). More recently, evidence showed that NT3 modulates the TrkC-mediated differentiation of excitatory presynaptic terminals (Ammendrup-Johnsen et al, 2015; Han et al, 2016). Therefore, the rescue of the impaired LTP in TgNTRK3 mice by exogenous application of NT3 might be contributed by a restored glutamatergic transmission. Supporting this hypothesis, we found that TgNTRK3 animals have an overinhibited infralimbic cortex due to a reduced presynaptic glutamate/GABA load (vGLUT1/vGAT puncta, data not shown), in agreement with the impaired LTP in the IL-mPFC. In fact, it has been shown that altered glutamatergic transmission is a neurochemical landmark in anxiety disorders, including PAND (Harvey and Shahid, 2012).

BDNF was shown to potentiate extinction memory when infused into the mPFC-IL (Peters et al, 2010). The authors showed that a flow of BDNF produced in the hippocampus to the mPFC-IL is necessary for successful fear extinction. We did not observe differences between WT and TgNTRK3 in hippocampal NT3 levels after fear extinction, suggesting that the NT3 deficit observed in the PFC of TgNTRK3 animals after fear is not due to a problem of NT3 production in the hippocampus.

In summary, we show that NT3/TrkC homeostasis is altered in the $\mathrm{mPFC}$ of $\operatorname{TgNTRK} 3$ mice and critically involved in fear extinction deficits in this mouse model of PAND (Dierssen et al, 2006; Santos et al, 2013). The impaired fear extinction is associated with a deficit of NT3 and plasticity in the mPFC-IL brain region, and is rescued by local NT3 infusion. Our study implicates for the first time NT3-induced synaptic plasticity in the modulation of pathological fear and thus identifies an entry site for the development of pharmacological support of cognitive behavioral therapy in PAND and other disorders of pathological fear. It needs to be determined whether the observed mechanisms are more generally involved in PAND and anxiety disorders of different etiology.

\section{FUNDING AND DISCLOSURE}

The authors declare no conflict of interest.

\section{ACKNOWLEDGMENTS}

The laboratory of MD is supported by DIUE de la Generalitat de Catalunya (Grups consolidats SGR 2014/1125). This work was supported by Fondation Jérôme Lejeune (Paris, France), MINECO (SAF2013-49129-C2-1-R and BFU2014-52467-R) and EU (Era Net Neuron PCIN-2013-060 and CORTICONIC 600806). The CRG is a Center of Excellence Severo Ochoa SEV-2012-0208. The CIBER of Rare Diseases is an initiative of the ISCIII. MS was supported by a postdoctoral fellowship by Fundação para a Ciência e Tecnologia (FCT, Portugal) and DD'A by La Caixa International PhD Programme fellowship.

\section{REFERENCES}

Aghajanian GK, Rasmussen K (1989). Intracellular studies in the facial nucleus illustrating a simple new method for obtaining viable motoneurons in adult rat brain slices. Synapse 3: 331-338.

Ammendrup-Johnsen I, Naito Y, Craig AM, Takahashi H (2015). Neurotrophin-3 Enhances the Synaptic Organizing Function of TrkC-Protein Tyrosine Phosphatase sigma in Rat Hippocampal Neurons. J Neurosci 35: 12425-12431.

Armengol L, Gratacos M, Pujana MA, Ribases M, Martin-Santos R, Estivill X (2002). 5' UTR-region SNP in the NTRK3 gene is associated with panic disorder. Mol Psychiatry 7: 928-930.

Collin C, Vicario-Abejon C, Rubio ME, Wenthold RJ, McKay RD, Segal M (2001). Neurotrophins act at presynaptic terminals to activate synapses among cultured hippocampal neurons. Eur J Neurosci 13: 1273-1282.

Dierssen M, Gratacos M, Sahun I, Martin M, Gallego X, AmadorArjona A et al (2006). Transgenic mice overexpressing the fulllength neurotrophin receptor TrkC exhibit increased catecholaminergic neuron density in specific brain areas and increased anxiety-like behavior and panic reaction. Neurobiol Dis 24: 403-418.

Feng G, Mellor RH, Bernstein M, Keller-Peck C, Nguyen QT, Wallace $M$ et al (2000). Imaging neuronal subsets in transgenic mice expressing multiple spectral variants of GFP. Neuron 28: 41-51.

Fischer A, Radulovic M, Schrick C, Sananbenesi F, GodovacZimmermann J, Radulovic J (2007). Hippocampal Mek/Erk signaling mediates extinction of contextual freezing behavior. Neurobiol Learn Mem 87: 149-158.

Gratacos M, Nadal M, Martin-Santos R, Pujana MA, Gago J, Peral B et al (2001). A polymorphic genomic duplication on human chromosome 15 is a susceptibility factor for panic and phobic disorders. Cell 106: 367-379.

Han KA, Woo D, Kim S, Choii G, Jeon S, Won SY et al (2016). Neurotrophin-3 Regulates Synapse Development by Modulating TrkC-PTPsigma Synaptic Adhesion and Intracellular Signaling Pathways. J Neurosci 36: 4816-4831.

Harvey BH, Shahid M (2012). Metabotropic and ionotropic glutamate receptors as neurobiological targets in anxiety and stress-related disorders: focus on pharmacology and preclinical translational models. Pharmacol Biochem Behav 100: 775-800.

Joo W, Hippenmeyer S, Luo L (2014). Neurodevelopment. Dendrite morphogenesis depends on relative levels of NT-3/TrkC signaling. Science 346: 626-629. 
Lessmann V (1998). Neurotrophin-dependent modulation of glutamatergic synaptic transmission in the mammalian CNS. Gen Pharmacol 31: 667-674.

Li R, Wu Y, Jiang D (2014). NT-3 attenuates the growth of human neuron cells through the ERK pathway. Cytotechnology 68: 659-664.

Lissek S, Powers AS, McClure EB, Phelps EA, Woldehawariat G, Grillon $C$ et al (2005). Classical fear conditioning in the anxiety disorders: a meta-analysis. Behav Res Ther 43: 1391-1424.

Lissek S, Rabin SJ, McDowell DJ, Dvir S, Bradford DE, Geraci M et al (2009). Impaired discriminative fear-conditioning resulting from elevated fear responding to learned safety cues among individuals with panic disorder. Behav Res Ther 47: 111-118.

Marek R, Coelho CM, Sullivan RK, Baker-Andresen D, Li X, Ratnu V et al (2011). Paradoxical enhancement of fear extinction memory and synaptic plasticity by inhibition of the histone acetyltransferase p300. J Neurosci 31: 7486-7491.

McAllister AK, Katz LC, Lo DC (1999). Neurotrophins and synaptic plasticity. Annu Rev Neurosci 22: 295-318.

Michael T, Blechert J, Vriends N, Margraf J, Wilhelm FH (2007). Fear conditioning in panic disorder: Enhanced resistance to extinction. J Abnorm Psychol 116: 612-617.

Milad MR, Quirk GJ (2002). Neurons in medial prefrontal cortex signal memory for fear extinction. Nature 420: 70-74.

Muinos-Gimeno M, Guidi M, Kagerbauer B, Martin-Santos R, Navines R, Alonso P et al (2009). Allele variants in functional MicroRNA target sites of the neurotrophin-3 receptor gene (NTRK3) as susceptibility factors for anxiety disorders. Hum Mutat 30: 1062-1071.

Parsons RG, Ressler KJ (2013). Implications of memory modulation for post-traumatic stress and fear disorders. Nat Neurosci 16: 146-153.

Paxinos G, Franklin KBJ. The mouse brain in stereotaxic coordinates. Academic Press: New York, 2001.

Peters J, Dieppa-Perea LM, Melendez LM, Quirk GJ (2010). Induction of fear extinction with hippocampalinfralimbic BDNF. Science 328: 1288-1290.
Quirk GJ, Mueller D (2008). Neural mechanisms of extinction learning and retrieval. Neuropsychopharmacology 33: $56-72$.

Reichardt LF (2006). Neurotrophin-regulated signalling pathways. Philos Trans $R$ Soc Lond B Biol Sci 361: 1545-1564.

Santini E, Ge H, Ren K, Pena de Ortiz S, Quirk GJ (2004). Consolidation of fear extinction requires protein synthesis in the medial prefrontal cortex. J Neurosci 24: 5704-5710.

Santos M, D'Amico D, Dierssen M (2015). From neural to genetic substrates of panic disorder: Insights from human and mouse studies. Eur J Pharmacol 759: 127-141.

Santos M, D'Amico D, Spadoni O, Amador-Arjona A, Stork O, Dierssen M (2013). Hippocampal hyperexcitability underlies enhanced fear memories in TgNTRK3, a panic disorder mouse model. J Neurosci 33: 15259-15271.

Sierra-Mercado D, Padilla-Coreano N, Quirk GJ (2011). Dissociable roles of prelimbic and infralimbic cortices, ventral hippocampus, and basolateral amygdala in the expression and extinction of conditioned fear. Neuropsychopharmacology 36: $529-538$.

Sotres-Bayon F, Bush DE, LeDoux JE (2007). Acquisition of fear extinction requires activation of NR2B-containing NMDA receptors in the lateral amygdala. Neuropsychopharmacology 32: 1929-1940.

Sotres-Bayon F, Quirk GJ (2010). Prefrontal control of fear: more than just extinction. Curr Opin Neurobiol 20: 231-235.

Takahashi H, Arstikaitis P, Prasad T, Bartlett TE, Wang YT, Murphy TH et al (2011). Postsynaptic TrkC and presynaptic PTPsigma function as a bidirectional excitatory synaptic organizing complex. Neuron 69: 287-303.

Tronson NC, Schrick C, Guzman YF, Huh KH, Srivastava DP, Penzes P et al (2009). Segregated populations of hippocampal principal CA1 neurons mediating conditioning and extinction of contextual fear. J Neurosci 29: 3387-3394.

Vidal-Gonzalez I, Vidal-Gonzalez B, Rauch SL, Quirk GJ (2006). Microstimulation reveals opposing influences of prelimbic and infralimbic cortex on the expression of conditioned fear. Learn Mem 13: 728-733. 